\title{
Noise Performance of the D0 Layer 0 Silicon Detector
}

\author{
M.. Johnson \\ Fermilab, MS 357, P.O. Box 500, Batavia, IL 60510-500 USA \\ E-mail address: mjohnson@fnal.gov
}

\begin{abstract}
A new inner detector called Layer 0 has been added to the existing silicon detector for the DZero colliding beams experiment. This detector has an all carbon fiber support structure that employs thin copper clad Kapton sheets embedded in the surface of the carbon fiber structure to improve the grounding of the structure and a readout system that fully isolates the local detector ground from the rest of the detector. Initial measurements show efficiencies greater than 90\% and 0.3 ADC count common mode contribution to the signal noise.
\end{abstract}

Keywords: Silicon Tracker, Carbon Fiber, Detector Grounding

\section{Introduction}

The original silicon detector for the D0 experiment had 4 layers of silicon. A new radiation hard inner layer (L0) was added to improve the resolution for displaced vertices and also provide increased redundancy for failures in the existing detector. Because the main barrel part of the existing detector could not be removed, this new detector had to be designed for in place insertion into the existing detector. This placed severe constraints on the design of the design. Table I shows the detailed specifications.

\section{TABLE I}

\begin{tabular}{|l|l|}
\hline Over all Length & $1660 \mathrm{~mm}$ \\
\hline Minimum diameter & $31.7 \mathrm{~mm}$ \\
\hline Sensor pitch & 71 or 81 micron \\
\hline Length of Sensors & 70 or $120 \mathrm{~mm}$ \\
\hline Number of sensors in longitudinal direction & 8 \\
\hline Number of sensors in azimuth direction & 6 \\
\hline Sensor thickness & $.3 \mathrm{~mm}$ \\
\hline
\end{tabular}

The overall length and small diameter required the use of very high modulus carbon fiber. It also made it very difficult to provide a dielectric break in the structure. High modulus carbon fiber is quite conductive at high frequencies so there is a classic ground loop formed by the grounded electronics at each end, the carbon fiber support tube and the rest of the detector. In order to eliminate this ground loop, we developed a readout system that isolates the local detector ground from the rest of the detector. We achieved isolation greater than10 ohms per end at $10 \mathrm{MHz}$. 
The small diameter of the detector did not allow the direct mounting of the readout chips on the sensors so we were forced to use a Kapton flex circuit cable (called the analog cable) between 200 and $360 \mathrm{~mm}$ long to bring the detector signal to the readout electronics. The capacitance of this cable $(.35 \mathrm{pF} / \mathrm{cm})$ doubled the detector capacitance, which then doubles the intrinsic noise of the detector.

\section{Mechanical Design}

The LØ support structure can be divided up into three major regions (fig. 1). The first region, occupying the central $760 \mathrm{~mm}$ of the structure, is the silicon sensor mounting outer shell with the precision sensor mounting surfaces. The second and third regions are the $450 \mathrm{~mm}$ long hexagonal hybrid mounting outer shells at each end of the structure. Cooling distribution manifold assemblies at each end also act as the support and connecting points for LØ. All of these components are connected together via a long dodecagonal inner shell.

The inner shell, the sensor mounting shell and the hybrid mounting shells are all made from unidirectional Mitsubishi Chemical K13C2U high modulus (900 GPa) carbon fiber pre-impregnated with RS-24 MOD resin supplied by YLA Inc. This pre-preg has a pre-cure fiber aerial weight of $66 \mathrm{~g} / \mathrm{m}^{2}$ and resin content of $39 \%$ by weight. The nominal thickness of the pre-preg is $63.5 \mu \mathrm{m}$. The inner shell spans the full length of the support structure $(1660 \mathrm{~mm})$ and has an inscribed diameter of $31.7 \mathrm{~mm}$. It is made of three layers of pre-preg with a $0^{\circ} / 90^{\circ} / 0^{\circ}$ arrangement. In another words, fibers are along the long axis in the two outside layers and are in the azimuth direction in the middle layer. The nominal cured thickness is $195 \mu \mathrm{m}$. The sensor mounting and hybrid mounting outer shells are both made of 5 layers of the same pre-preg with a symmetrical fiber orientation of $+20^{\circ} \%-20^{\circ} / 0^{\circ} /-20^{\circ} /+20^{\circ}$. An outer layer of Kapton ${ }^{\circledR}$ with copper mesh and gold plated contacts is co-cured with the outer shells giving a combined cured thickness is $325 \mu \mathrm{m}$. The sensor mounting outer shell has a six-sided castellated shape that allows for mounting of the silicon sensors at two different radial locations.

A series of copper ground circuits printed on flexible Kapton film $(0.025 \mathrm{~mm}$ thick) are applied to the outer layer of carbon fiber on the sensor mounting surfaces, and are bonded to the shell during the cure process. The copper mesh is facing the carbon fiber. This process forced the copper mesh into good electrical contact with the conductive carbon fibers. These Kapton sheets are installed using the same techniques used for the plies of pre-preg with special care required to ensure that the Kapton/Cumesh conformed to the surface features of the castellated shell with no corner air gaps. Contact to the copper mesh is made by plated through holes in the Kapton and gold pads on the outer surface. For the B-layer sensors that mount on the top of the castellations a flexible grounding strap is bonded to the ground circuit on the bottom of the castellation to make contact with the underside of the sensor.

\section{Electrical Design}

The electrical design was dominated by the need to eliminate common mode noise. Because of the added capacitance of the analog cable and the use of $300 \mu$ thick detectors, even a small amount of common mode noise could seriously affect the 
performance of the detector. In addition the analog cable can act as a good antenna for picking up common mode noise

The overall philosophy is two fold. First, we isolate the detector grounds from the rest of the world to prevent any ground loops through the body of the detector. Second, we provide low impedance connections between the sensor and the SVX 4 chip [1] so that there is little relative voltage between them at all frequencies in the pass band of the SVX 4.

Fig. 2 shows a simplified schematic of the detector readout electronics. Every circuit has an input path and a return path. The input path to the SVX 4 is from the reversed bias diode through the analog cable and into the preamp in the SVX 4. There are no local ground traces on the analog cable and the cables are separated by a $200 \mu$ polypropylene spacer that is needed to minimize cable-to-cable capacitance. Because there is no ground plane, detector pulses will couple into neighboring channels. However, the time constants for the adjacent channels are much smaller than the integration time for the SVX 4 so almost all the charge flows back into main channel before the end of the charge integration time. There is a trade off between the capacitance between the traces in the cable and the ground plane and the loop area between the traces and the ground plane. The larger the spacing the smaller the capacitance but the loop area increases which may pick up noise. In this design we emphasized smaller capacitance.

The return circuit to the bias side of the silicon diode is as important as the input circuit. The return side of the silicon diode is at the bias voltage so the bias voltage must be connected to the amplifier ground. In the layer 0 design this is done at both the hybrid and the sensor.

The bias voltage is transmitted from the hybrid to the sensor over a trace at the edge of the analog cable. The return current flows on an adjacent trace. These traces have a resistance of $20 \mathrm{ohms}$ and are also quite inductive. This impedance is too large for the traces to be a good path for the return current. Because of this, the capacitor at the hybrid is not very important for the return current. We put a large series resistance in the bias supply line upstream of the hybrid so the hybrid capacitor is part of an RC filter for the bias voltage. We chose instead to use the carbon fiber structure of the detector itself as the ground return. Not only did this allow for much lower impedance, but it also forced the structure to be at the same voltage as the SVX 4 ground so that there would be little or no capacitive coupling into the analog cable.

As discussed in [2], carbon fiber is quite conductive at high frequencies but it is not conductive enough to provide a low impedance path. We increased the conductivity of the carbon fiber by placing a sheet of copper clad Kapton ( $25 \mu$ thick Kapton coated with a $5 \mu$ thick layer of copper) on the surface of the carbon fiber structure. The Kapton is etched in a mesh pattern (234 $\mu$ trace width, $1.45 \mathrm{~mm}$ pitch, 30\% copper coverage) to reduce the amount of added material. The mesh size is very small so for frequencies that the SVX 4 can respond to it appears as a solid sheet of copper.

The connections shown in fig. 3 from the hybrid to the Kapton sheet and from the Kapton sheet to the filter board on the sensor are quite important. They must be low inductance and have a very small loop area. The Beryllium Oxide hybrid is 380 microns thick and has a gold plated stripe on one side. This stripe is connected to the hybrid ground plane by plated through holes in the $\mathrm{BeO}$. The Kapton mesh on the carbon fiber 
support structure has a corresponding gold plated section. The hybrid is then glued to this support structure with conductive epoxy. This creates a connection with very low inductance and almost no loop area. The outer layer hybrids are connected to the ground plane by wide copper clad Kapton tabs.

Each of the two SVX 4 chips on a hybrid was connected to the hybrid ground plane with a single point ground. This was done to minimize ground currents inside the chip. All the SVX 4 ground bonds are connected to a pad that is the size of the SVX 4 chip. This pad is connected to the hybrid ground plane at one point.

A small printed circuit board is mounted on top of the sensor. This card receives the bias voltage from the analog cable and has a capacitor to ground. Since the main return path is through this capacitor, its connection to the carbon fiber is very important. We used copper plated Kapton tabs, which were connected to gold plated pads on the copper ground mesh with conductive epoxy (fig. 3).

The ground loop through the body of the detector is eliminated by creating an isolated ground on the detector side of the electronics. We do not need large voltage isolation so we chose a fairly simple method. We converted all single ended signals to differential ones and sent the signals across the ground barrier to a differential receiver. The power supplies use local regulators that were chosen on the basis of their AC isolation specifications. However, the isolation was not adequate so we added additional differential and common mode filtering outside the detector.

On an individual basis these ideas worked quite well. But there are about 500 parallel connections at each end so the overall ground isolation is about 10 ohms at 10 $\mathrm{MHz}$ which is adequate for this device.

The power supplies need a DC reference point so that the power supply voltages have the same 0 point on both ends. This is provided through the high voltage bias system. The bias voltage return line has a $10 \mathrm{~K}$ ohm resistor to external ground that provides both ground isolation and the proper DC reference point.

\section{Noise Calculations}

We define the noise on the output of channel $n$ as the standard deviation of the digital output in ADC counts for that channel:

$$
\sigma_{a}^{2}=\overline{a_{n}^{2}}-{\overline{a_{n}}}^{2}
$$

We also define "differential" noise on a given channel as

$$
\left(\sigma_{a}^{\text {diff }}\right)^{2}=\frac{\overline{\left(a_{n+1}-a_{n}\right)^{2}}-\overline{\left(a_{n+1}-a_{n}\right)^{2}}}{2}
$$

$\sigma_{a}^{\text {diff }}$ is estimated by computing the root mean square value of 
over a large number of events.

$$
\frac{a_{n+1}-a_{n}}{\sqrt{2}}
$$

Differential noise would be a good estimator of common mode noise if there were no correlations between adjacent channels. However, adjacent channels are correlated both through the interstrip capacitance and the capacitance between traces in the analog cable. Following [3] we calculate (2). The total noise charge, $Q_{n}$, in channel $n$ is

$$
\begin{aligned}
Q_{n} & =C_{s}\left(U_{n-1}-\left(2 C_{s}+C_{g}+C_{A}\right) U_{n}+C_{s} U_{n+1}\right. \\
& =C_{s}\left(U_{n-1}-(2+r) U_{n}+U_{n+1}\right)
\end{aligned}
$$

where $\mathrm{U}$ is the noise amplitude, $C_{s}$ is the capacitance between adjacent channels including the analog cable, $C_{g}$ is the capacitance to ground, $C_{A}$ is the total amplifier capacitance and $r=\left(C_{q}+C_{A}\right) / C_{s}$. Next, form the ratio of the differential noise to the noise in a single channel:

$$
\frac{\left(Q_{n+1}-Q_{n}\right) / 2}{Q_{n}}=\frac{\left.\left.\left(U_{n-1}-(3+r) U_{n}\right)+(3+r) U_{n+1}-U_{n+2}\right)\right) / 2}{U_{n-1}-(2+r) U_{n}+U_{n+1}}
$$

Assuming that all the noise amplitudes are identical and converting to expectation values we get:

$$
\frac{\left\langle\left(Q_{n+1}-Q_{n}\right) / 2\right\rangle^{2}}{\left\langle Q_{n}\right\rangle^{2}}=\frac{10+6 r+r^{2}}{6+4 r+r^{2}}
$$

$C_{A}$ is estimated [3] from the noise performance of the SVX $4(380+41$ electrons $/ \mathrm{pF}$ ) to be $9.3 \mathrm{pF}$. From sensor and analog cable measurements, $C_{s}=11 p F$ and $C_{g}=1 p F$ giving $r=0.93$ and

$$
\left\langle Q_{n}\right\rangle=.8\left\langle\left(Q_{n+1}-Q_{n}\right) / 2\right\rangle
$$

That is, we would expect the single channel noise to be $80 \%$ of the differential noise. If the single channel noise is larger than this, there is a contribution form common mode noise.

\section{Performance}


Fig. 4 shows the pedestal (blue), 10 times the total noise (cyan) and 10 times the differential noise (yellow) for the entire layer 0 detector in normal running mode. The average differential noise is about 1.8 and the average total noise is about 1.7 ADC counts. From (7) we would expect the total noise to be 1.4 ADC counts so we conclude that there are about 0.3 ADC counts of common mode noise.

We also connected the detector ground to the isolated ground and measured the change in noise. There was only a small increase which indicates that the major contribution to the low noise is from the overall grounding of the detector.

\section{References}

[1] B. Krieger et al.,IEEE Trans. Nucl. Sci. 51., 1968 (2004)

[2] W. Cooper et al.,Nucl. Instrum. Meth. A 550, 127 (2005)

[3] G. Lutz, Nucl. Instrum. Meth. A 309, 545 (1991)

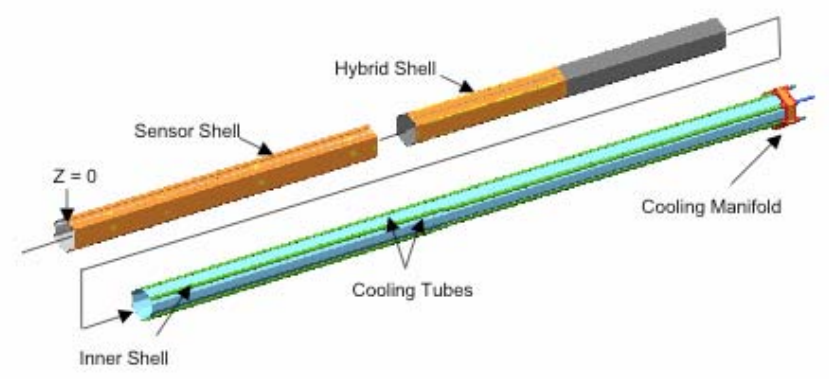

Fig.1.Drawing of the layer 0 detector showing the sensor mounting shell, the hybrid mounting shell and the inner support shell. 


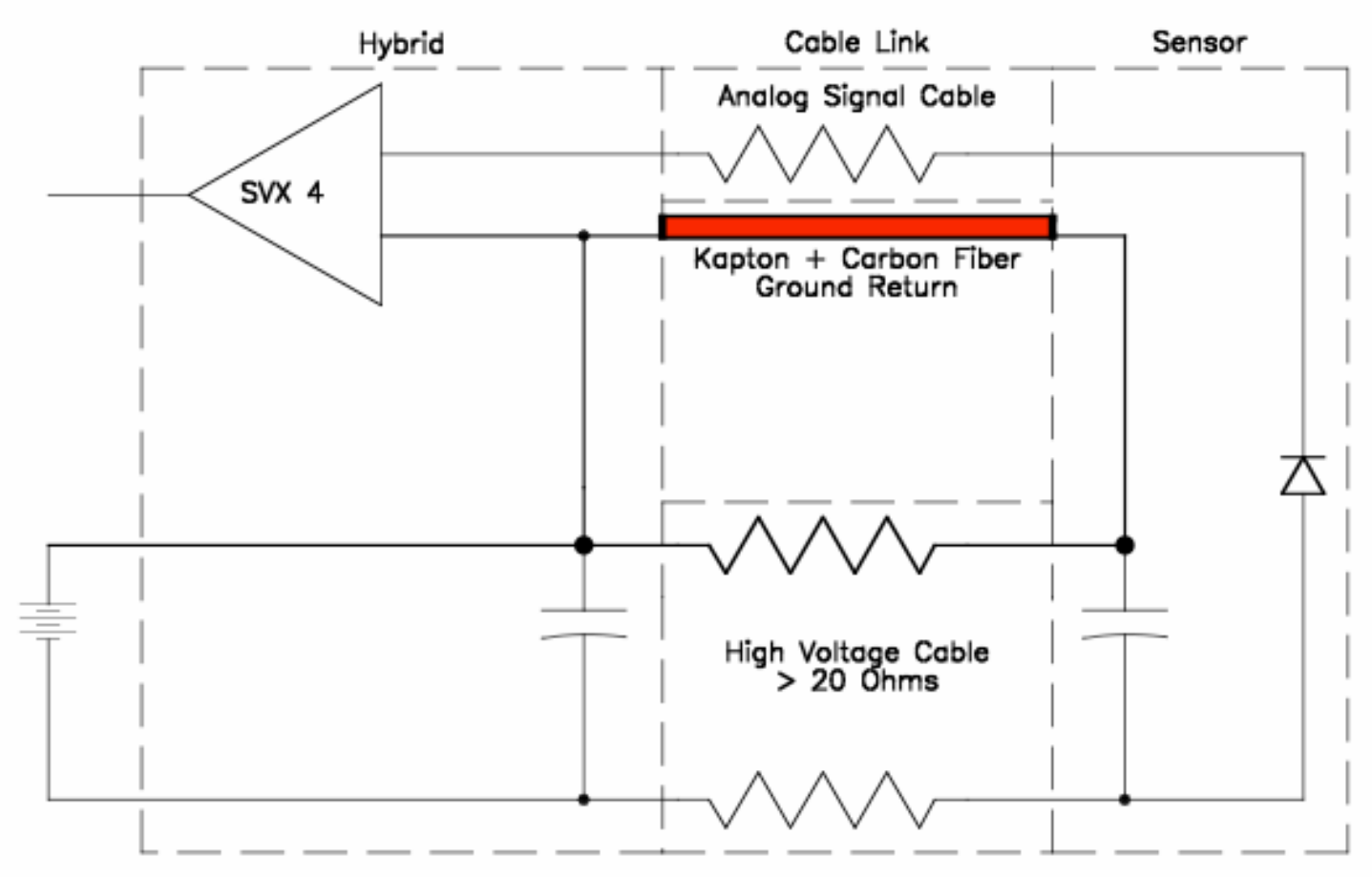

Fig. 2. Simplified schematic of the layer 0 electrical circuit. The hybrid refers to the $\mathrm{BeO}$ hybrid mounted on the carbon fiber substrate, the link is the combination of the analog cable and the carbon fiber support structure and the sensor includes the bias filter board. 


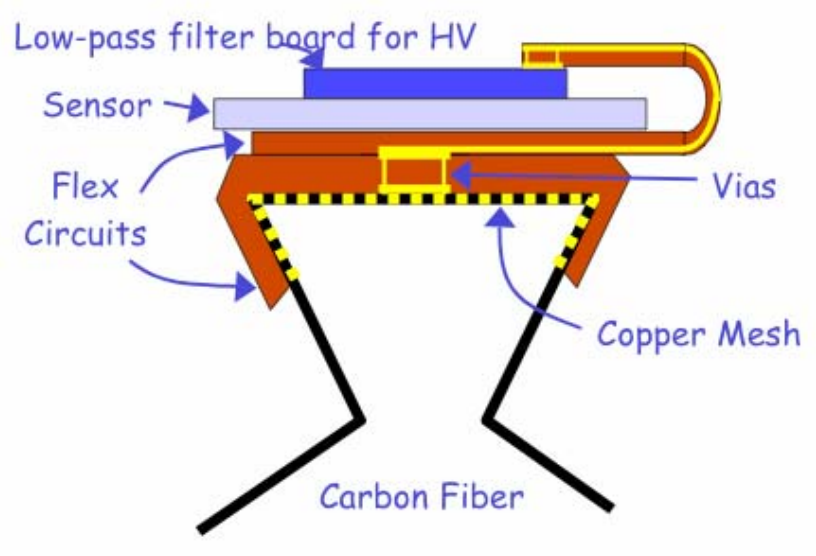

Fig. 3. Sketch of copper tab connection between the ground plane and the bias voltage board. 


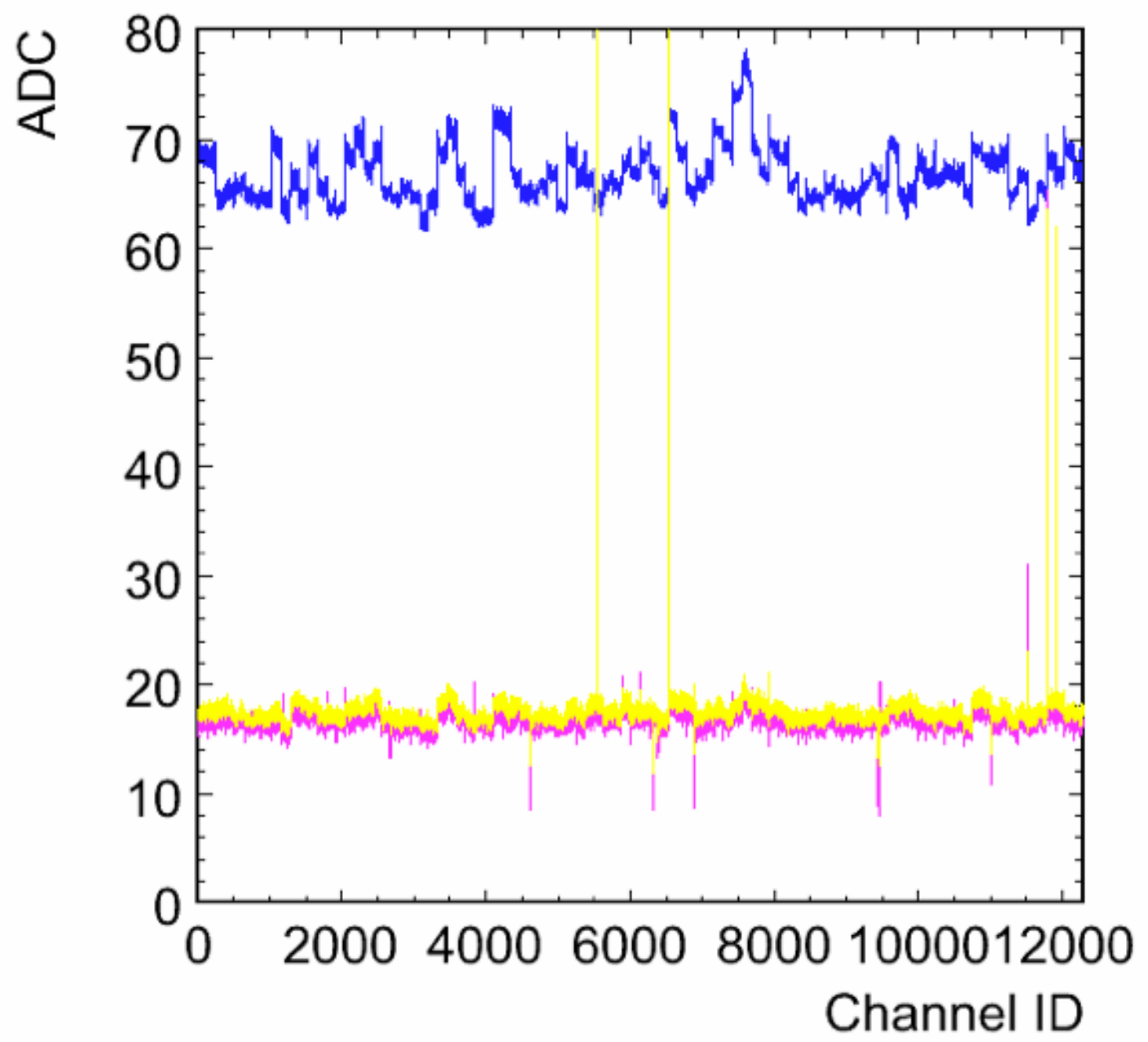

Fig. 4. Pedestal and noise signal for 1 sector of layer 0 . The blue trace is the average pedestal. The yellow trace is 10 times the sigma for that channel and the purple trace is 10 times the differential noise (see text). 